\title{
Novice motor performance: Better not to verbalize
}

\author{
Guillaume Chauvel • François Maquestiaux • \\ Eric Ruthruff • André Didierjean • Alan A. Hartley
}

Published online: 17 October 2012

(C) Psychonomic Society, Inc. 2012

\begin{abstract}
Offline verbalization about a new motor experience is often assumed to positively influence subsequent performance. Here, we evaluated this presumed positive influence and whether it originates from declarative or from procedural knowledge using the explicit/implicit motorlearning paradigm. To this end, 80 nongolfers learned to perform a golf-putting task with high error rates (i.e., explicit motor learning), and thus relied on declarative knowledge, or low error rates (i.e., implicit motor learning), and thus relied on procedural knowledge. Afterward, they either put their memories of the previous motor experience into words or completed an irrelevant verbal task. Finally, they performed the putting task again. Verbalization did not improve novice motor performance: Putting was impaired, overall, and especially so for high-error learners. We conclude that declarative knowledge is altered by verbalization, whereas procedural knowledge is not.
\end{abstract}

G. Chauvel $\cdot$ F. Maquestiaux $(\bowtie)$

Université Paris-Sud, UFR STAPS,

Bât 335,

91405 Orsay Cedex, France

e-mail: francois.maquestiaux@u-psud.fr

G. Chauvel

e-mail: guillaume.chauvel@u-psud.fr

E. Ruthruff

University of New Mexico,

Albuquerque, New Mexico, USA

A. Didierjean

Université de Franche-Comté \& Institut Universitaire de France,

Besançon, France

A. A. Hartley

Scripps College,

Claremont, CA, USA
Keywords Novice motor performance · Verbalization · Declarative knowledge $\cdot$ Procedural knowledge $\cdot$ Skill acquisition $\cdot$ Language/memory interactions

In the fields of sports, it is often assumed that verbally expressing one's recent motor action helps novices to efficiently learn to perform a new motor skill. French legislation even explicitly states that high-school students should acquire verbal knowledge about how to perform motor skills that are taught in physical education classes (Programme d'éducation physique et sportive, 2010). In any case, it is quite common for novices to verbalize when learning a new sport (or when learning other motor routines, such as those related to driving or manufacturing). But does verbalization actually help?

In a pioneering study on verbalization and motor performance, Flegal and Anderson (2008) observed that, among skilled golfers, offline verbalization had no positive influence: Those with a moderate level of expertise putted equally well after describing their memory of the motor skill, and those with an intermediate level performed worse. It is highly plausible, however, that novice golfers, who have not yet automatized their performance, would actually benefit from verbalization (as is assumed by French legislation and many sports teachers). The present study addressed the novel issue of how verbalization influences novice motor performance, using an approach similar to Flegal and Anderson's. This issue is important, since all players begin as novices, and any method that improves initial learning might place the individuals on a higher learning trajectory, with long-lasting consequences. We also examined how verbalizing influences the different types of memory representations developed at the outset of skill acquisition. 


\section{Previous studies of verbalization in perceptual and motor tasks}

The hypothesis that offline verbalization impairs memory has received some empirical support from studies using perceptual tasks. Schooler and Engstler-Schooler (1990) found that verbally describing a nonverbal stimulus, such as a face, can impair its subsequent recognition. These authors were the first to hypothesize that verbalization creates a language-based representation, which overshadows difficult-to-verbalize aspects of the perceptual memory that are important for effective recognition. This negative effect of verbalization on memory was termed the verbalovershadowing effect. This effect has been observed in other perceptual domains, such as describing a picture of a mushroom (Melcher \& Schooler, 2004), describing a previously studied route map (Fiore \& Schooler, 2002), or wine tasting (Melcher \& Schooler, 1996).

When easy-to-verbalize tasks are used, such as recalling a spoken word (Schooler \& Engstler-Schooler, 1990) or solving a logic problem (Schooler, Ohlsson, \& Brooks, 1993), verbalization does not hinder, and sometimes even improves, memory performance. Therefore, one boundary condition for observing verbal-overshadowing is the use of difficult-to-verbalize tasks. Another boundary condition is an excess of perceptual expertise relative to verbal expertise. For instance, Melcher and Schooler (1996) found that verbalization altered recognition of a previously sampled wine among individuals with an intermediate skill level (hypothesized to have a high level of perceptual expertise relative to their verbal expertise), whereas it had no significant effect among novices (who have similarly low levels of perceptual and verbal expertise) or expert wine drinkers (who have similarly high levels of perceptual and verbal expertise). Additionally, verbalization impaired later recognition of a target mushroom only among the participants who had previously received perceptual training to recognize mushrooms (Melcher \& Schooler, 2004).

To assess whether the verbal-overshadowing effect also applies to motor performance, Flegal and Anderson (2008) employed a golf-putting task performed $183 \mathrm{~cm}$ from the hole. Such a motor task complies well with the first boundary condition for observing verbal overshadowing, being difficult to put into words. To comply with the second boundary condition, Flegal and Anderson recruited 40 golfers with a moderate level of skill expertise (about 6 years' golfing, mean self-reported score of 54.6 on a nine-hole course) and 40 golfers with an intermediate level of skill expertise (almost 9 years' golfing, mean self-reported score of 42.5). According to motor-skill acquisition theory (e.g., Fitts \& Posner, 1967), knowledge representations that are generally accessible to conscious report (i.e., declarative knowledge) are mainly involved at low levels of expertise, while knowledge representations that generally are not open to conscious report (i.e., procedural knowledge) become increasingly important as expertise develops. Following this theory, Flegal and Anderson assumed that the excess of procedural expertise over verbal expertise was especially pronounced for more skilled golfers.

In Flegal and Anderson's (2008) procedure, the skilled golfers were required to achieve a criterion of three consecutive successful putts. They were then asked, for a period of $5 \mathrm{~min}$, either to verbalize what they remembered from their previous motor experience or to perform an irrelevant verbal task. Afterward, they were again required to reach the criterion of three putts. The data showed that verbalization increased the number of putts necessary to reachieve the performance criterion for more skilled golfers, while it had no significant effect for moderately skilled golfers. In fact, the more skilled golfers who verbalized needed twice as many putts to reachieve the criterion as did those who had not verbalized.

One post hoc explanation offered by Flegal and Anderson (2008) to account for the negative effect of verbalization was an extension of the verbal-overshadowing effect (Schooler, 2002) to the motor domain. Specifically, vigorously engaging declarative memory temporarily disrupts the use of stored procedural learning, which is more dominant in the motor control of golfers with an intermediate skill level. Broadly consistent with this view, verbalization may induce enduring changes to the procedural representation under lying the motor skill by decoupling it into its constituent parts (Masters, 1992). Another post hoc account offered by Flegal and Anderson proposed that verbalizing causes a residual bias of attention to the verbal elements of the skill when later performing it, potentially raising the likelihood that conscious control will interfere with motor performance that runs better automatically (for a review consistent with this proposal, see Masters \& Maxwell, 2008).

\section{The effects of online attention on motor performance: A different issue}

In previous research examining how attention influences motor skill execution, it has been consistently observed that attending online to components of a sports-related task has, in novices, either no effect (Gray, 2004) or some positive effects (Beilock, Carr, MacMahon, \& Starkes, 2002), but in experts it has negative effects (Beilock et al., 2002; Gray, 2004; Jackson, Ashford, \& Norsworthy, 2006). Although these studies of attention during skill execution and Flegal and Anderson's (2008) study of verbalization following skill execution may resemble each other, there are two major differences between them. First, the manipulation aimed at 
influencing motor behavior was online in the previous studies, but offline in Flegal and Anderson's study. Second, attending to movement in the previous studies was not necessarily a verbal act: It might merely reflect an attempt to control movement. In Flegal and Anderson's study, however, the act was undoubtedly verbal, since participants had to translate into words their previous motor experiences. As a consequence, the literature examining how online attention influences movement says little, if anything, about how offline verbalization of skill knowledge might influence subsequent motor performance.

\section{The present study: Goals and predictions}

Flegal and Anderson's (2008) pioneering study demonstrated that offline verbalization does not improve subsequent motor performance, and may in fact impair it, at least in a sample of skilled individuals. However, a different outcome might be expected with golf novices - verbalization might improve their subsequent motor performance-given that they do not exactly meet the two boundary conditions stated above for observing verbal overshadowing. Therefore, the goal of the present study was to determine whether verbalization really improves novice motor performance. We are aware of no previous studies that have directly addressed this issue.

We also examined the effects of verbalization on declarative and procedural memory representations separately, using a manipulation that induced predominant use of one type of knowledge over the other; novice golfers were ideal participants for such a manipulation because, by definition, they had little or no prior task knowledge. Research initiated by Masters (1992; for a review, see Masters \& Poolton, 2012) has shown that the use of these two forms of knowledge in motor performance can be modified by the manner in which people learn a skill. Maxwell, Masters, Kerr, and Weedon (2001), for example, demonstrated that explicit-learning conditions that promote high error rates cause learners to actively test hypotheses about how best to move in order to avoid errors, which results in the accretion of a high preponderance of declarative knowledge. Alternatively, learning conditions that promote low error rates cause learners to test hypotheses about their movements less actively, which results in a lower preponderance of declarative knowledge.

Maxwell et al. (2001) reported two critical indicators that the type of knowledge accumulated during learning was indeed influenced by error rates. First, an attentiondemanding secondary task (tone counting), performed simultaneously with the golf-putting task, disrupted motor performance for high-error but not for low-error learners. Second, hypothesis testing during learning, associated with declarative knowledge, was greater in high-error that in low- error learners. In sum, declarative knowledge was more influential in high-error learners' performance, whereas procedural knowledge was more influential in low-error learners' performance (for corroborating evidence, see Chauvel et al., 2012; for reviews of other relevant studies, see Chauvel et al., 2011; Masters \& Poolton, 2012).

Using the protocol developed by Maxwell et al. (2001) and Chauvel et al. (2012), we trained novice golfers to putt explicitly (to produce a large amount of declarative knowledge) or implicitly (to produce a small amount of declarative knowledge). Half of the learners performed blocks of putts farther from the hole, thus producing a low percentage of successful putts (i.e., the high-error learners); the other half performed blocks of putts at distances near the hole, thus producing a high percentage of successful putts (i.e., the low-error learners). After learning, a randomly selected half of the low-error and high-error learners verbalized their memories of the putting task (the verbalizers). The other half were required to perform an irrelevant verbal task (the nonverbalizers). In the transfer phase, all of the participants performed the golf-putting task at the novel distance of $125 \mathrm{~cm}$, exactly in-between the distances used by the lowand high-error groups during training. Note that, in contrast to Maxwell et al. and Chauvel et al. (2012), participants did not perform a tone-counting task.

On the basis of a common assumption from the fields of sports and physical education, it can be predicted that verbalization improves procedural and/or declarative knowledge, leading to motor improvement at transfer for at least one of the two learning groups. In contrast, the verbal-overshadowing literature raises the possibility that verbalizing a previous motor experience will impair subsequent performance. The most natural expectation is that impairment would be most severe for those relying primarily on procedural memory (the low-error learners), for instance because verbalization might induce greater reliance on declarative memory. For those relying on declarative memory (the high-error learners), verbalization might merely help them to consolidate their declarative knowledge. However, this hypothesis is speculative and clearly needs empirical support to back it up.

\section{Method}

\section{Participants}

A group of 80 adults (age: $M=24.1$ years, $S D=4.4$ years, range $=18-35$ years; 38 women, 42 men) with no previous golfing experience volunteered from the local community of greater Paris. They gave their written informed consent before participation. All of the participants were treated within the ethical guidelines of the American Psychological Association. 


\section{Apparatus}

Participants attempted putts to a hole $11.4 \mathrm{~cm}$ in diameter (regulation size for golf) on a level artificial-turf indoor green $(200 \times 270 \mathrm{~cm})$ raised $15 \mathrm{~cm}$ above ground level. A collecting duct was fitted beneath the hole. Standard white golf balls were used. Identical right- and left-handed (lengths of 87 or $90 \mathrm{~cm}$ ) putters were available to suit each participant's preference.

\section{Procedure}

In the learning phase, the high-error participants putted from distances of 225, 200, 175, and $150 \mathrm{~cm}$ (in that order), and the low-error participants putted from distances of 25, 50, 75 , and $100 \mathrm{~cm}$ (in that order). Forty putts were completed in each block before switching to the next distance. Participants were instructed to putt as many balls as possible into the hole at their own pace (less than 5 min was generally sufficient to complete each block). Learning blocks were interspersed with 3-min breaks during which participants played the number game Sudoku (i.e., a task unrelated to golf putting, thus preventing them from thinking about their previous experience). Then, half of both the high-error and low-error participants were randomly assigned to the verbalization group. They spent $3 \mathrm{~min}$ writing a detailed description of how they had previously performed the task. Each of the verbalizers received the following instructions: "Remember everything on which your attention was focused during the learning phase, whether it was before, during, or after each putt. Think about each of the 160 trials." The other half of the high-error and low-error participants were assigned to the no-verbalization group. These nonverbalizers spent 3 min performing word search games with words unrelated to golf putting. In the transfer phase, all of the participants performed one block of 40 trials at the novel distance of $125 \mathrm{~cm}$.

\section{Overview of the main analyses}

To assess golf-putting performance during learning, an analysis of variance (ANOVA) was conducted on the mean numbers of successful putts, with type of verbalization group (verbalization or no verbalization) as a betweensubjects variable and putting distance (1st, 2nd, 3rd, or 4th block) as a within-subjects variable. This ANOVA was carried out separately for each learning group because the putting distances were different. Independent-samples $t$ tests were carried out on the amount and the quality of verbalization reported to have been used during learning by the verbalizers, with type of learning group as a betweensubjects variable. Golf-putting performance during transfer was assessed using ANOVAs carried out separately for each learning group on the mean numbers of successful putts, with type of verbalization group as a between-subjects variable and putting distance (4th learning block or transfer block) as a within-subjects variable. None of the participants were excluded from the statistical analyses.

\section{Results}

Figure 1 shows the performance for each group of participants during learning and transfer.

\section{Learning phase}

The number of successful putts steadily decreased as the putting distance from the hole increased for the low-error participants, $F(3,114)=64.45, p<.001$, and steadily
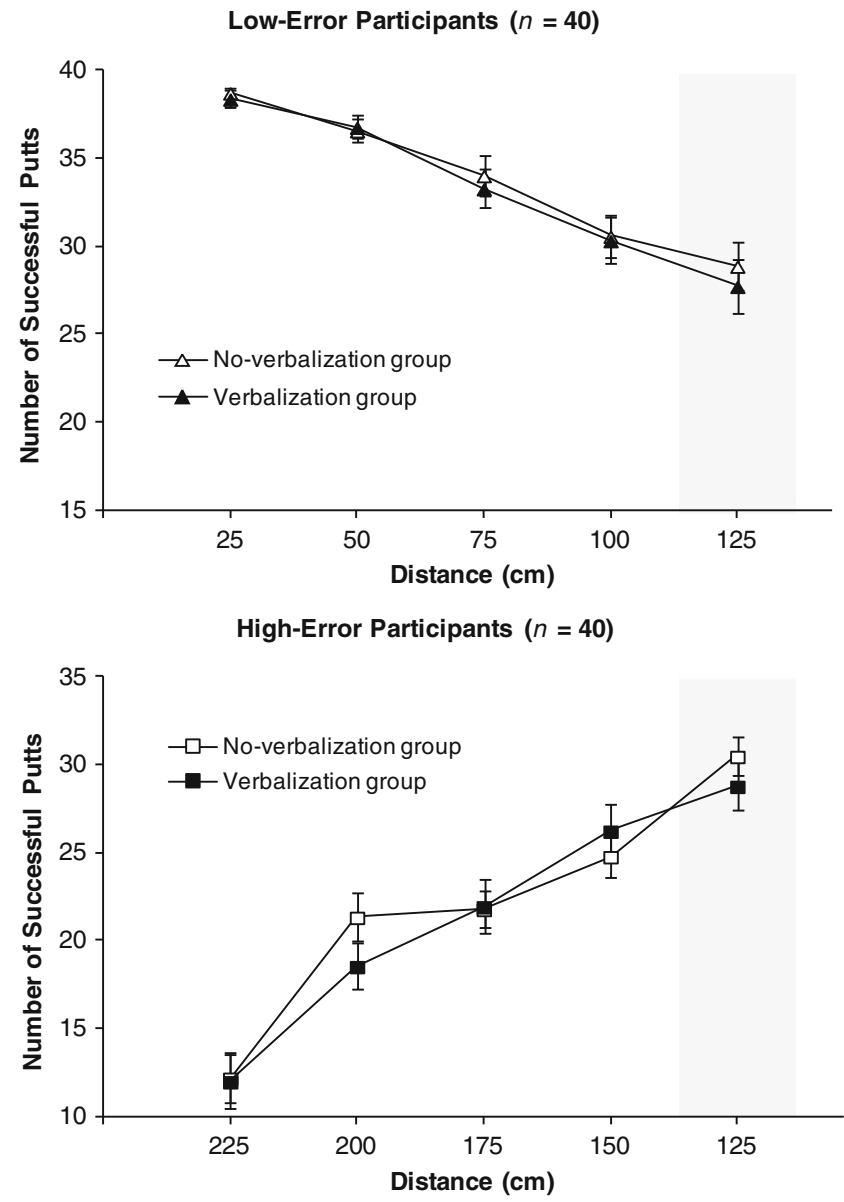

Fig. 1 Mean numbers of successful putts (out of 40) as a function of distance from the hole in the learning phase (unshaded area) and the transfer phase (shaded area) for low-error and high-error participants. The half of participants who verbalized their previous learning experience (between the last learning block and the transfer block) are represented by filled symbols, while the half who performed an irrelevant verbal task are represented by unfilled symbols. The bars show the standard errors for each putting distance 
increased as the putting distance decreased for the high-error participants, $F(3,114)=109.02, p<.001$. The large difference in the overall percentages of successful putts between low-error participants (87.1\% success) and high-error participants (49.7 \% success) was significant, $t(78)=15.17$, $p<.001$, indicating that the manipulation of motor experience type (low vs. high error) was successful.

In each learning group, the number of successful putts was obviously uninfluenced by whether or not the participants would later go on to verbalize, $F \mathrm{~s}<1$. The type of verbalization group did not interact with putting distance for the low-error participants, $F<1$. Surprisingly, however, it did interact with putting distance for the high-error participants, $F(3,114)=2.69, p=.049$. To disentangle this interaction, we performed separate ANOVAs for verbalizers and nonverbalizers, followed by post hoc comparisons using the Bonferroni procedure. These analyses indicated that this interaction was due to the absence of a difference, solely for the nonverbalizers, between motor performance at the distances of $200 \mathrm{~cm}(M=21.4, S D=6.2)$ and $175 \mathrm{~cm}$ $(M=21.8, S D=4.8)$, as well as at the distances of 175 and $150 \mathrm{~cm}(M=24.8, S D=5.4)$. We have no good explanation for these performance fluctuations limited only to one of the four subgroups of participants.

\section{Verbalization phase}

The numbers of words written during the 3-min verbalization period were comparable for the low-error participants $(M=51.7, S D=17.5)$ and the high-error participants $(M=54.7, S D=22.4), t(38)<1$. The numbers of putting steps described, as determined by averaging the counts from two independent raters [interrater reliability was significant: $r(40)=.60, p<.001]$, were also comparable for the lowerror participants $(M=2.3, S D=1.6)$ and the high-error participants $(M=2.4, S D=1.4), t(38)<1$. As in Maxwell et al. (2001) and Chauvel et al. (2012), independent raters also assessed the numbers of statements indicative of the use of hypothesis-testing strategies while putting [interrater reliability was significant: $r(40)=.68, p<.001]$. The number of strategies reported by verbalizers was greater for high-error participants $(M=2.4, S D=.96)$ than for low-error participants $(M=1.7, S D=.86), t(38)=2.25, p<.05$. This finding suggests that the memory representations underlying the putting behavior differed between the two learning groups.

\section{Transfer phase}

We tested for verbalization effects at transfer by comparing performance changes between the last learning block and the transfer block, separately for each learning group. For low-error participants, the number of successful putts decreased by 2.1 on average from the last learning distance of
$100 \mathrm{~cm}(M=30.4, S D=5.6)$ to the transfer distance of $125 \mathrm{~cm}(M=28.3, S D=6.5), F(1,38)=8.64, p<.01$. The decrease was slightly larger for verbalizers (decrease of 2.6 putts, from 30.4 to 27.8 ) than for nonverbalizers (decrease of 1.7 putts, from 30.6 to 28.9), though the interaction did not reach statistical significance, $F(1,38)<1$. Although the data did lean toward a small negative effect of verbalization on putting, we were unable to detect it as significant, even with a sample of 40 low-error participants.

For high-error participants, the number of successful putts increased from the last learning distance of $150 \mathrm{~cm}$ $(M=25.5, S D=6.0)$ to the transfer distance of $125 \mathrm{~cm}$ $(M=29.7, S D=5.4), F(1,38)=37.86, p<.001$. Critically, this effect interacted significantly with verbalization, $F(1,38)=$ $5.52, p<.05$. For the nonverbalizers, the number of successful putts significantly increased by 5.5 putts on average, from $150 \mathrm{~cm}(M=24.8, S D=5.4)$ to $125 \mathrm{~cm}(M=30.5, S D=$ 4.7), $t(38)=3.55, p<.01$. For the verbalizers, however, the numbers of successful putts did not differ significantly between $150 \mathrm{~cm}(M=26.3, S D=6.6)$ and $125 \mathrm{~cm}(M=28.8, S D=5.9)$, $t(38)=1.29, p=.21$. In sum, verbalization disrupted motor performance for the high-error participants.

Because the two groups had putted from different distances prior to transfer, a special procedure was required to directly compare their verbalization effects. The key was the observation that, for both learning groups, performance improved approximately linearly as the distance from the hole decreased (see Fig. 1). Accordingly, the numbers of successful putts were regressed onto the four learning distances for each individual, and the resulting best-fitting equation was used to predict each individual's performance at the transfer distance of $125 \mathrm{~cm}$. We then computed the percentage deviation between the predicted and observed numbers of successful putts at $125 \mathrm{~cm}$.

The factorial ANOVA revealed that the percentage deviation was $-6.09 \%$ for verbalizers but $2.14 \%$ for nonverbalizers, a marginally significant difference, $F(1,76)=3.35$, $p=.07$. This trend is consistent with an overall cost of verbalization. However, the cost of verbalization did not interact significantly with type of learning, $F(1,76)<1$. Among low-error participants, the percentage deviations did not differ significantly between the verbalizers $(M=-1.92)$ and the nonverbalizers $(M=2.19), t(38)<1$. Among higherror participants, the percentage deviation was $-10.25 \%$ for the verbalizers but was $2.10 \%$ for the nonverbalizers; this difference also did not reach statistical significance, $t(38)=1.83, p=.08$.

\section{Discussion}

In the present study, we assessed whether, among novice individuals, thinking about a previous motor experience 
positively influenced subsequent performance. Flegal and Anderson's (2008) pioneering study of verbalization and movement demonstrated no positive effect among skilled individuals; however, one might have expected to find a real benefit of verbalization for novices. The use of novice participants also allowed us to determine which type of knowledge (procedural vs. declarative) is most sensitive to the effects of verbalization. Novices have, by definition, no prior knowledge of how to perform a skill, so it is relatively easy to manipulate the type of knowledge (declarative vs. procedural) developed at the outset of skill acquisition by using the explicit/implicit motor-learning paradigm (e.g., Maxwell et al., 2001).

Overall, the present data were at odds with the view that sport novices should verbalize their motor performance. Verbalizing a previous motor experience did not benefit subsequent performance for novice participants, regardless of the learning type (high or low error). In fact, the higherror participants showed significant impairment following verbalization and, averaged across both groups, the trend toward impairment was marginally significant.

Interestingly, these data are inconsistent with the most natural expectations based on the verbal-overshadowing literature (Schooler, 2002), as applied to the motor domain (Flegal \& Anderson, 2008). The performance of low-error learners, who are presumably more influenced by nonverbal procedural knowledge, should have been the most disrupted by verbalization. Yet, if anything, the opposite effect occurred. One post-hoc explanation is that verbalization distorted declarative knowledge representations (e.g., by overwriting or editing them) but left intact procedural representations, without actually shifting the reliance on procedural versus declarative knowledge. As a consequence, the low-error participants had distorted declarative knowledge but still relied on procedural knowledge, while the higherror participants continued to use declarative knowledge even though it was distorted.

Our findings are consistent with those of Liao and Masters (2001, Study 2) who showed that motor performance was influenced by a prior thought suppression intervention ("try not to think about the rules of topspin hitting"), which ironically aimed at producing the opposite effect (i.e., inducing participants to think about the rules). The thought suppression manipulation impaired the motor performance of participants who learned the table tennis task explicitly (via instructions), whereas it did not affect the performance of participants who learned the task implicitly (using an analogy-learning paradigm that limited the development of declarative knowledge).

In summary, the present study extended the findings of Flegal and Anderson (2008) from skilled participants to novice participants: Even briefly verbalizing one's motor skill after execution can have detrimental effects on later execution. In particular, we found that verbalizing novice motor performance for 3 min provoked enduring changes for the novices, especially in those individuals presumed to predominantly rely on declarative knowledge. We can safely conclude that verbalizing a motor skill can change the course and structure of cognitive processes, potentially leading to poorer subsequent motor performance (for a similar proposal with regard to cognitive tasks, see Ericsson \& Simon, 1980). A practical implication of the present findings is that promoting the use of verbalization as a means of improving motor learning is unnecessary (at best), or even hazardous (at worst).

Author Note We thank Rich Masters for his commentary and insights.

\section{References}

Beilock, S. L., Carr, T. H., MacMahon, C., \& Starkes, J. L. (2002). When paying attention becomes counterproductive: Impact of divided versus skill-focused attention on novice and experienced performance of sensorimotor skills. Journal of Experimental Psychology: Applied, 8, 6-16. doi:10.1037/1076-898X.8.1.6

Chauvel, G., Maquestiaux, F., Didierjean, A., Joubert, S., Dieudonné, B., \& Verny, M. (2011). Use of nondeclarative and automatic memory processes in motor learning: How to mitigate the effects of aging. Gériatrie et Psychologie Neuropsychologie du Vieillissement, 9, 455-463.

Chauvel, G., Maquestiaux, F., Hartley, A. A., Joubert, S., Didierjean, A., \& Masters, R. W. S. (2012). Age effects shrink when motor learning is predominantly supported by nondeclarative, automatic memory processes: Evidence from golf putting. Quarterly Journal of Experimental Psychology, 65, 25-38. doi:10.1080/17470218.2011.588714

Ericsson, K. A., \& Simon, H. A. (1980). Verbal reports as data. Psychological Review, 87, 215-251. doi:10.1037/0033-295X.87.3.215

Fiore, S. M., \& Schooler, J. W. (2002). How did you get here from there? Verbal overshadowing of spatial mental models. Applied Cognitive Psychology, 16, 897-910.

Fitts, P. M., \& Posner, M. I. (1967). Human performance. Belmont, CA: Brooks/Cole.

Flegal, K. E., \& Anderson, M. C. (2008). Overthinking skilled motor performance: Or why those who teach can't do. Psychonomic Bulletin \& Review, 15, 927-932. doi:10.3758/PBR.15.5.927

Gray, R. (2004). Attending to the execution of a complex sensorimotor skill: Expertise differences, choking, and slumps. Journal of Experimental Psychology: Applied, 10, 42-54.

Jackson, R. C., Ashford, K. J., \& Norsworthy, G. (2006). Attentional focus, dispositional reinvestment, and skilled motor performance under pressure. Journal of Sport and Exercise Psychology, 28, 49-68.

Liao, C.-M., \& Masters, R. S. W. (2001). Analogy learning: A means to implicit motor learning. Journal of Sport Sciences, 19, 307-319.

Masters, R. S. W. (1992). Knowledge, knerves, and know-how: The role of explicit versus implicit knowledge in the breakdown of a complex motor skill under pressure. British Journal of Psychology, 83, 343-358.

Masters, R. S. W., \& Maxwell, J. (2008). The theory of reinvestment. International Review of Sport and Exercise Psychology, 1, 160-183. 
Masters, R. S. W., \& Poolton, J. M. (2012). Advances in implicit motor learning. In N. J. Hodges \& A. M. Williams (Eds.), Skill acquisition in sport: Research, theory and practice (2nd ed., pp. 59-75). London: Routledge.

Maxwell, J. P., Masters, R. S. W., Kerr, E., \& Weedon, E. (2001). The implicit benefit of learning without errors. Quarterly Journal of Experimental Psychology, 54A, 1049-1068.

Melcher, J. M., \& Schooler, J. W. (1996). The misremembrance of wines past: Verbal and perceptual expertise differentially mediate verbal overshadowing of taste memory. Journal of Memory and Language, 35, 231-245.

Melcher, J. M., \& Schooler, J. W. (2004). Perceptual and conceptual training mediate the verbal overshadowing effect in an unfamiliar domain. Memory \& Cognition, 32, 618-631. doi:10.3758/BF03195853
Programme d'éducation physique et sportive pour les lycées d'enseignement général et technologique. (2010, April 29). Bulletin Officiel, 4, 1-14.

Schooler, J. W. (2002). Verbalization produces a transfer inappropriate processing shift. Applied Cognitive Psychology, 16, 989-997.

Schooler, J. W., \& Engstler-Schooler, T. Y. (1990). Verbal overshadowing of visual memories: Some things are better left unsaid. Cognitive Psychology, 22, 36-71. doi:10.1016/00100285(90)90003-M

Schooler, J. W., Ohlsson, S., \& Brooks, K. (1993). Thoughts beyond words: When language overshadows insight. Journal of Experimental Psychology: General, 122, 166-183. doi:10.1037/00963445.122.2.166 